\title{
Opto-Mechanical Modal Spectroscopy: Opto-Excited Vibrations of a Micron-Scale On-Chip Resonator
}

Tal Carmon and Kerry J. Vahala

Caltech, 1200 E. California BLVD. Pasadena CA 91125.

tal@caltech.edu,www.vahala.caltech.edu

\begin{abstract}
Radiation pressure is used to excite vibrational modes of an optical micro-cavity at $\mathrm{GHz}$ rates. Many spectral lines associated with high-order vibrational modes are measured. Perturbation of the cavity geometry is observed to induce splitting of the spectral lines. (C) 2007 Optical Society of America

OCIS codes: (230.1040) Acousto-optical devices; (300.6330) Spectroscopy, inelastic scattering including Raman.
\end{abstract}

Radiation pressure (RP) can push the boundaries of an optical cavity to change its optical path length [1, 2]. In doing this, RP also couples the optical and mechanical motion of the cavity. Recently, this coupling was used to excite regenerative mechanical vibrations below $100 \mathrm{MHz}$ in a microtoroidal resonator [3-5]. The underlying physics of these RP-coupled optical and mechanical oscillators has applications to both classical and quantum phenomena [2,6,7]. Generally, in quantum opto-mechanical applications, it is preferable for the mechanical-oscillation quanta, $\hbar \Omega$, to be as large as possible when compared with the thermal noise quantum, $K_{b} T$.

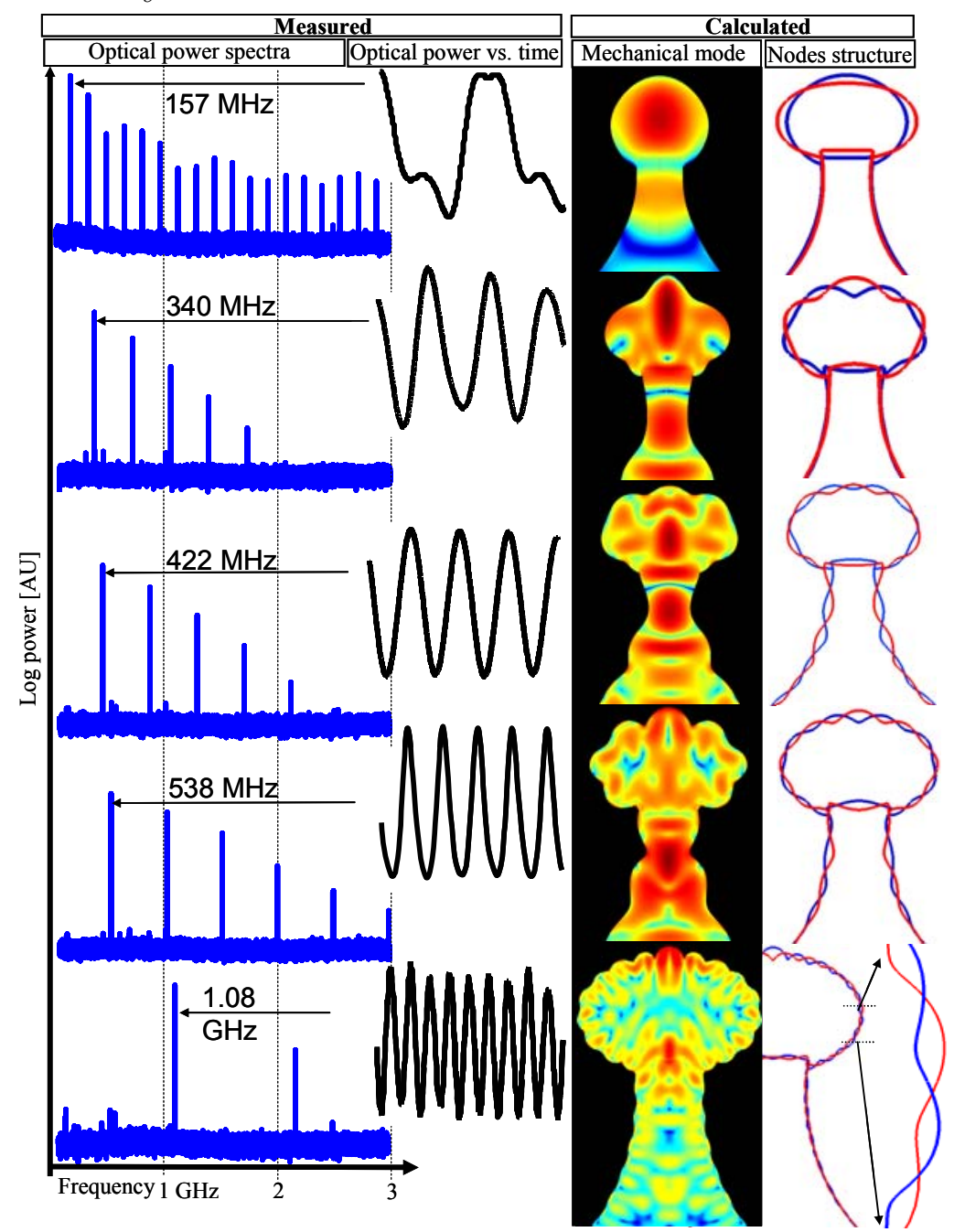

Figure 1: Measured optical-power oscillation with the corresponding calculated mechanical modes where colors represent log of the strain energy. Also calculated is the nodes geometry. The drawn deflection here is exaggerated. 


\section{JMB2.pdf}

Here we demonstrate RP excitation of the high-order mechanical modes of a spherical micro cavity. Vibrations at frequencies above $1 \mathrm{GHz}$ are excited in a $24 \mu \mathrm{m}$ silica spheroid [8] on a silicon-chip platform. The simple experimental setup consists of a $\mathrm{CW}$ optical input coupled to a spherical cavity by means of tapered fiber [9]. Radiation pressure created by the light circulating in the cavity excites vibrational modes of the sphere via a parametric instability as described in refs [3-5] for the case of toroidal devices. It is emphasized that the optical imput here is $\mathrm{CW}$ and that no external modulation or feedback is used. Exciting high-order mechanical modes is done by shortening the lifetime of the optical-cavity photons, which, in practice, is done by changing the coupling. In figure 1, the measured spectral lines are plotted next to the calculated eigen mechanical modes, and the agreement of the calculated and measured modes is within 3\%. Of significance is the excitation of a vibrational mode with eigenfrequency $1.08 \mathrm{Ghz}$ (fig 1 bottom). The theoretical strain field for this mode shows that it contains many nodes. Another aspect of this form of spectroscopy is the relation between cavity perturbation and fine splitting of the spectral line. For this purpose, we fabricated a toroidal cavity with an eccentric boundary. A calculation (fig 2 a) reveals two eigen mechanical modes for this eccentric resonator that are rotated with respect to each other. A $0.25 \mathrm{MHz}$ splitting occurs between these respectively-rotated modes as a result of the perturbation. A corresponding splitting of the spectral lines is also observed in fig $2 \mathrm{~b}$.

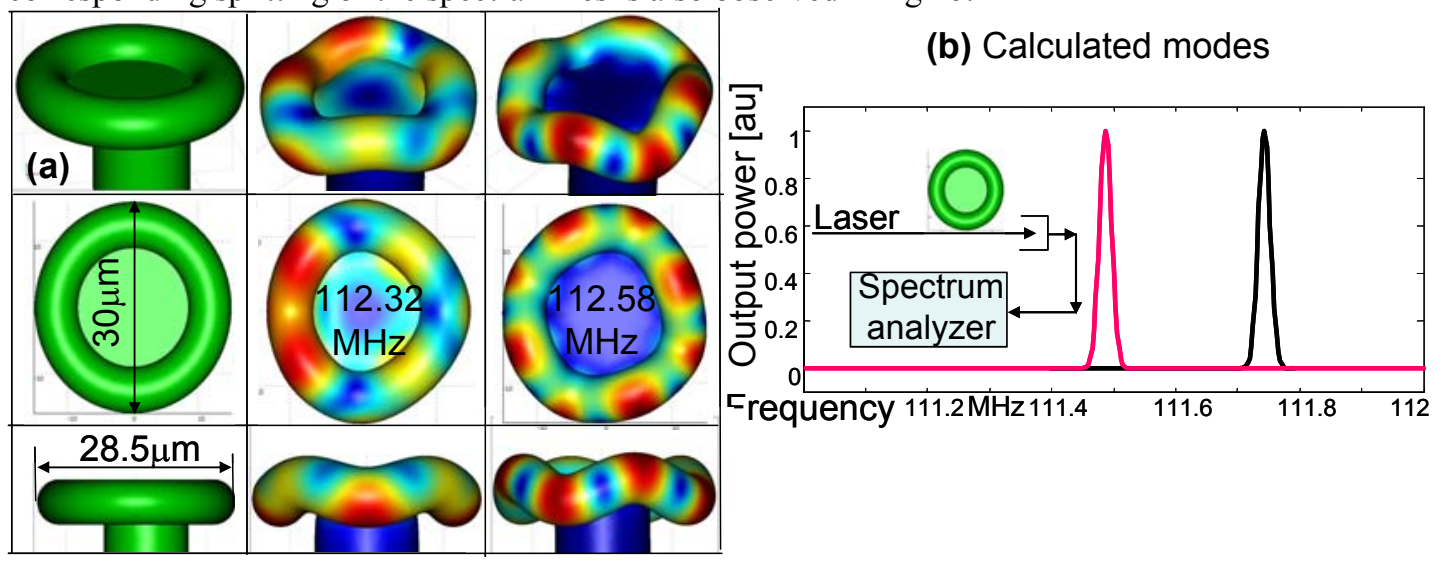

Figure 2: =.(a) Calculation shows the split into two degenerated modes oriented with different rotation.

(b) Experimental spectrum of an anisotropic toroidal cavity reveals spectral line splitting (red and black lines).

Color represents deformation. Deformation is exaggerated in figures.

To conclude, in analogy to optical spectroscopy as a tool to deduce the mechanical modes of a molecule, we have here studied the spectroscopy associated with the mechanical modes of a macroscopic object by measuring its interaction with radiation. Many spectral lines related to high-order modes are excited and perturbations are measured to give rise to spectral splitting. Additionally, the high frequencies presented here are attractive for experiments in quantum opto-mechanics.

[1] A. Dorsel, J.D. McCullen, P. Meystre, E. Vignes \& H. Walther, "Optical Bistability and Mirror Confinement Induced by Radiation Pressure," Phys Rev Lett 51, 1550 (1983).

[2] V.B. Braginsky, S.E. Strigin \& S.P. Vyatchanin, "Parametric oscillatory instability in Fabry-Perot interferometer," Physics Letters A 287, 331 (2001).

[3] T. Carmon, H. Rokhsari, L. Yang, T.J. Kippenberg \& K.J. Vahala, "Temporal behavior of radiation-pressure-induced vibrations of an optical microcavity phonon mode," Phys Rev Lett 94, 223902 (2005).

[4] H. Rokhsari, T.J. Kippenberg, T. Carmon \& K.J. Vahala, "Radiation-pressure-driven micro-mechanical oscillator," Opt Express 13, 5293 (2005).

[5] T.J. Kippenberg, H. Rokhsari, T. Carmon, A. Scherer \& K.J. Vahala, "Analysis of radiation-pressure induced mechanical oscillation of an optical microcavity," Phys Rev Lett 95, 033901 (2005).

[6] W. Marshall, C. Simon, R. Penrose \& D. Bouwmeester, "Towards quantum superpositions of a mirror," Phys Rev Lett 91, $130401(2003)$.

[7] S. Mancini, D. Vitali \& P. Tombesi, "Scheme for teleportation of quantum states onto a mechanical resonator," Phys Rev Lett 90, $137901(2003)$.

[8] D.K. Armani, T.J. Kippenberg, S.M. Spillane \& K.J. Vahala, "Ultra-high-Q toroid microcavity on a chip," Nature 421, 925 (2003).

[9] M. Cai, O. Painter \& K.J. Vahala, "Observation of critical coupling in a fiber taper to a silica-microsphere whispering-gallery mode system," Phys Rev Lett 85, 74 (2000). 